\title{
Building from the Blueprint for Patient Safety at the Hospital for Sick Children
}

$\overline{\text { Polly Stevens, Anne Matlow and Ronald Laxer }}$

\section{INTRODUCTION}

The Hospital for Sick Children (Sick Kids) has an international reputation as a leader in children's healthcare. Our own experience, however, and evidence on the prevalence of error in healthcare has shown us that even the best, all too often, make mistakes. In 2002, responding to a compelling goal to make our hospital safer for the vulnerable children and families who turn to us for care, we launched the Blueprint for Patient Safety (Blueprint), a comprehensive and action-oriented plan. The plan has been updated annually and is currently on its fourth iteration. This article will touch on the 10 components of the plan, focusing on specific initiatives and lessons we have learned while using the Blueprint to build our patient safety program and develop a culture of safety within the organization.

\section{External Review and Key CONCEPTS}

In 2002, when the Blueprint was being drafted, there were no regulatory nor accreditation requirements to guide us in its development. A review of the literature and best practices of local and international organizations yielded a number of concepts that informed our work. These are included in Table 1.
Table 1. List of key concepts and sources

\begin{tabular}{ll}
\hline $\begin{array}{l}\text { Concept } \\
\text { The "systems" approach }\end{array}$ & Sample source \\
“Just" culture & Reason 1997 and 2000 \\
Complexity theory and complex & Marx 2001 \\
$\quad$ adaptive systems & Zimmerman et al. 1998 \\
High reliability organizations & \\
Hindsight bias & Weick and Sutcliffe 2001 \\
Human factors engineering & Bogner 1994 \\
"Extreme" honesty and humanistic & Vincent 2001 \\
\hline
\end{tabular}

risk management

Table 2. Factors affecting implementation of the plan

\begin{tabular}{ll}
$\begin{array}{l}\text { Facilitating factors } \\
\text { A “burning platform” following two } \\
\text { very tragic adverse events }\end{array}$ & $\begin{array}{l}\text { Challenging factors } \\
\text { Fear of reprisal }\end{array}$ \\
\hline Strong leadership support & $\begin{array}{l}\text { Strong program and professional } \\
\text { autonomy }\end{array}$ \\
\hline $\begin{array}{l}\text { Good quality improvement culture } \\
\text { and infrastructure }\end{array}$ & $\begin{array}{l}\text { Inconsistent follow-up of ideas } \\
\text { and initiatives }\end{array}$ \\
\hline $\begin{array}{l}\text { Well established morbidity and } \\
\text { review processes }\end{array}$ & $\begin{array}{l}\text { Fragmented risk-reporting mortality } \\
\text { systems }\end{array}$ \\
\hline $\begin{array}{l}\text { Clinical information systems with } \\
\text { electronic order set capability }\end{array}$ & Fragmented clinical information \\
\hline Committed teams & systems \\
\hline $\begin{array}{l}\text { Compelling mission to improve } \\
\text { health of children }\end{array}$ & Relatively young workforce \\
\hline
\end{tabular}




\section{Organizational Assessment}

An internal review highlighted factors that we felt would facilitate implementation of the plan and ones that might make it more challenging. These are included in Table 2. Facilitating factors are included in Table 2 , and challenging factors are included in Table 3.

Table 3. Examples of improvements resulting from critical occurrence reviews

Implementation of a transfer checklist to improve communication at hand-overs

Comprehensive rare test proficiency testing

Standardization and controls in the use of heparin

Changes in the air traffic control of helicopters landing on the hospital's roof

Changes to consent processes

Changes to blood bank processes

Removal of concentrated potassium chloride from nursing units

Changes in equipment cleaning

Tighter clinic referral processes

Development of controls in the management of expressed breast milk

Standardization of correct site/procedure processes

\section{Quality versus Patient Safety Concept}

In order to build on a culture of quality improvement that was already well established in the organization, a graphic was developed to help teach staff the relationship between quality improvement and patient safety (Figure 1). The left side of the graphic depicts 10 children who have come to the hospital for treatment with each experiencing a specific level of care ranging from low to high quality. The middle section depicts the focus of quality improvement, which is to raise the ceiling so that

Figure 1. Relationship between quality improvement and patient safety

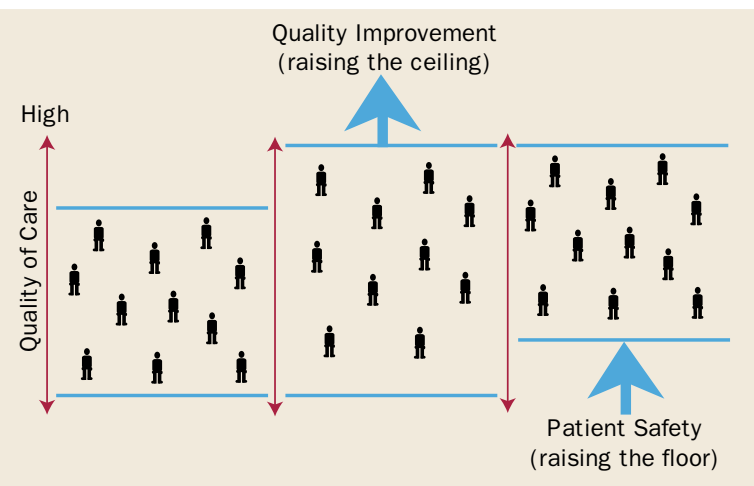

higher levels of care can be achieved. The far right section depicts the focus of patient safety, which is to raise the floor so that fewer patients experience poor levels of care or are harmed. Ultimately, both quality improvement and patient safety work together to improve the overall quality of care provided.

\section{Program Model and Components}

In order to quickly communicate the elements of the patient safety plan, a simple model was developed (Figure 2) to highlight the need for integrating external and internal patient safety information, for identifying vulnerabilities and unsafe practices, and for making and evaluating appropriate change. The model also emphasizes the overarching role of leadership, culture and communication.

Figure 2. Patient safety plan - conceptual framework

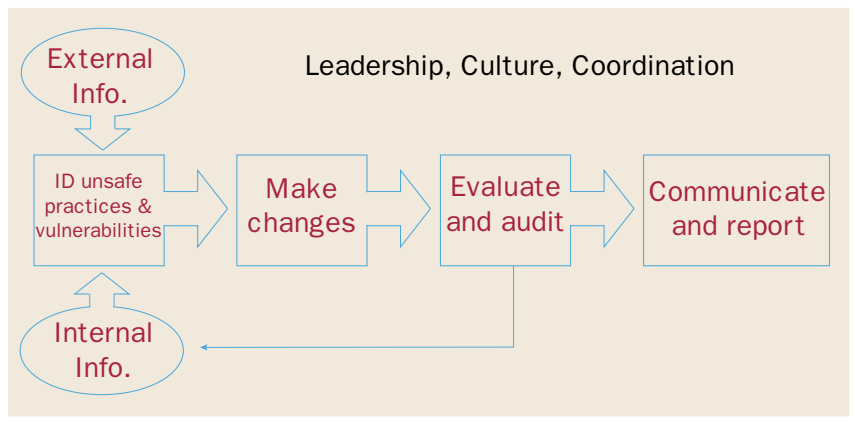

\section{COMPONENTS}

The Blueprint is divided into 10 components:

1. Leadership and Culture

2. Management of Critical Occurrences

3. External Surveillance

4. Internal Surveillance

5. Policies, Procedures and Guidelines

6. Staff Education and Partnerships

7. Partnering with Patients and Families

8. Program Coordination

9. Proactive Risk Assessment and Audit

10. Evaluation and Research

Each of these components will be briefly discussed, focusing on actions, results and lessons learned.

\section{Leadership and Culture}

The first component represents the most critical element of the plan - leadership and culture. An organization's leaders are essential in advancing any new agenda. They set goals and establish priorities, develop plans and allocate resources. Just as important, they create a culture that promotes new initiatives by 
articulating shared values, modeling appropriate behaviours and establishing expectations for staff. An organization committed to patient safety articulates this as an essential organizational goal and then translates this goal into specific actions (Kohn et al. 2000). Specific activities related to this component include ensuring that patient safety is included in the strategic plan, the annual goals and objectives, and in the operating plan and budget; as well, patient safety should be addressed at the time of hire and be part of regular performance reviews.

Patient safety has been firmly entrenched in organizational strategy and operations, and there are numerous examples of the organization putting the safety of children before other competing priorities, which include: the development of a preoperative anesthesia clinic to better screen high-risk children before surgery; the purchase of state-of-the-art paediatric compatible, physiological monitoring equipment; and, despite an earlier focus on same-day admissions for surgery, recognizing that for some children, admission the night before provides an essential safety net to ensure they are properly prepared for surgery.

In November 2004, safety "walkarounds" were initiated, in which a member of the hospital executive and others meet with staff on a clinical unit and discuss patient safety and environmental safety concerns. Action items, responsibilities and feedback are essential components of the process. So far, common themes arising from the sessions include bed management, communication, equipment, staffing and patient transfers. A subsequent evaluation of the rounds has resulted in a guideline document to improve the operational and follow-up elements of the walkarounds; as well, a database has been developed to facilitate tracking of feedback and actions. Plans are in place to extend the walkarounds to non-clinical areas.

\section{Management of Critical Occurrences}

Unfortunately, despite our best intentions, errors happen that result in significant harm to patients. It is important that senior leadership, medical staff and employees handle these events with courage and honesty, and with a commitment to finding and improving system issues and to sharing these lessons with others. At Sick Kids, a policy and guideline for managing critical occurrences has been developed and includes immediate patient care and family considerations, support of staff involved in events and processes for investigating the event, developing recommendations and for ensuring appropriate follow-up of changes. A companion policy on disclosure highlights the patient's and family's right to be informed following an adverse event and provides direction for staff in managing these discussions.

Both policies are frequently requested by other organizations, and an article highlighting the unique aspects of disclosure in a paediatric institution was published in the Journal of Pediatrics (Matlow et al. 2004). The hospital has also been asked to provide expert testimony on our management of critical occur- rences processes and provide advice to colleagues managing critical events in other organizations. Perhaps though, the most meaningful endorsement of our approach came following the inquest into the tragic post-operative death of a young patient at our hospital. One of the recommendations contained in the final report was the following:

We, the Jury recommend that, for health care, the systems approach to patient safety be adopted...We endorse and encourage the use of the systems approach as adopted by The Hospital for Sick Children as a means of enhancing patient safety. (Chief Coroner of Ontario, 2002).

A primary focus of our critical occurrence reviews is the development of recommendations for improving the system and for preventing the recurrence of similar events. Some of the improvements that have been initiated following a critical occurrence review are listed in Table 3.

One of the challenges we have faced as a result of doing comprehensive reviews is ensuring that recommendations resulting from the reviews are implemented and have the intended effect. This was easier to do when the process began. However, with the hundreds of recommendations that have been generated to date, follow-up has become a significant challenge. We have recently developed an electronic database to facilitate tracking and trending of quality and risk management information, including recommendations from critical occurrence reviews.

\section{External Surveillance}

Thankfully, we do not have to experience harmful adverse events to learn from them. By making an effort to learn from the mistakes of others we have the opportunity to improve care without the human toll associated with an actual event. Recently, there has been an explosion of information on patient safety. Literature, conferences, agencies and networks abound that promote a greater understanding of medical error and communicate "best practices" in patient safety. With the amount of information that is available to us, we run the risk of either spending too much time in reviewing marginal material or failing to note information that could benefit the organization. As a result, we required a coordinated system for reviewing external information, evaluating its usefulness and ensuring the appropriate implementation of recognized safe practices.

At Sick Kids, we have created an inventory of external data sources, which we routinely survey for relevant safety information. In September 2004, a system was implemented to manage and track hazard alerts and recall information, primarily related to medical devices, supplies and medications. This entailed a database linked to our e-mail system, in which hazard or recall information is entered and appropriate individuals are identified 
for follow-up. This information is conveyed through e-mail, and the subsequent responses are recorded for review by the Hazard Alerts Committee. To date, almost 600 entries have been made into the system (an average of 60/ month), and the response rate of notified individuals within an appropriate window of time has improved to almost $100 \%$ (up from $50 \%$ prior to the change).

\section{Internal Surveillance}

Improvements in patient safety require a comprehensive understanding of what is going on in the organization, including the incidence of error, harm and potential harm. Studies have shown that incidents are vastly under reported by staff; however, reporting rates can be improved when (1) staff feel safe in reporting events, (2) when reports are easy to complete and (3) when staff receive feedback about positive changes that were made as a result of this information (Zipperer and Cushman 2001). Emphasis on the reporting of potential incidents and close calls is also essential as it allows for learning without the challenges associated with actual events.

At Sick Kids, there were a number of different errorreporting systems (including patient and visitor, medication, staff and critical care), which resulted in some confusion at the front lines. Paper-based reporting led to delays in notification and follow-up, manual data entry was required to identify trends, and in some areas, incident reports were being used to evaluate staff performance.

In May 2004, a comprehensive, on-line safety reporting system for all events was launched with the goals of increasing the number of reports, increasing the proportion of potential or near-miss reports,, improving turn-around time for report follow-up and making improvements in patient safety. Anonymous reporting was provided as an option. In the 12 months since the system has been operational, we have seen a $60 \%$ increase in the number of reports and a similar increase in the proportion of potential reports (Figures 3 and 4). About 16\% of reports are entered anonymously, a number we would like to see decrease as staff learn to trust the system. However, our goal of increasing timeliness of report follow-up has not yet been achieved. We have found that with both the increase in the volume of reports and more onus on front-line managers to manage reports, timeliness of follow-up remains about the same as it was prior to the new system. Our plan to provide regular, area-specific indicators on outstanding reports is expected to help focus accountability on this important aspect of the system.

A number of hospital-wide projects have been initiated as a result of safety reporting, including improvements to bed safety, entanglement and patient identification.
Figure 3. Safety reporting system results - total reports

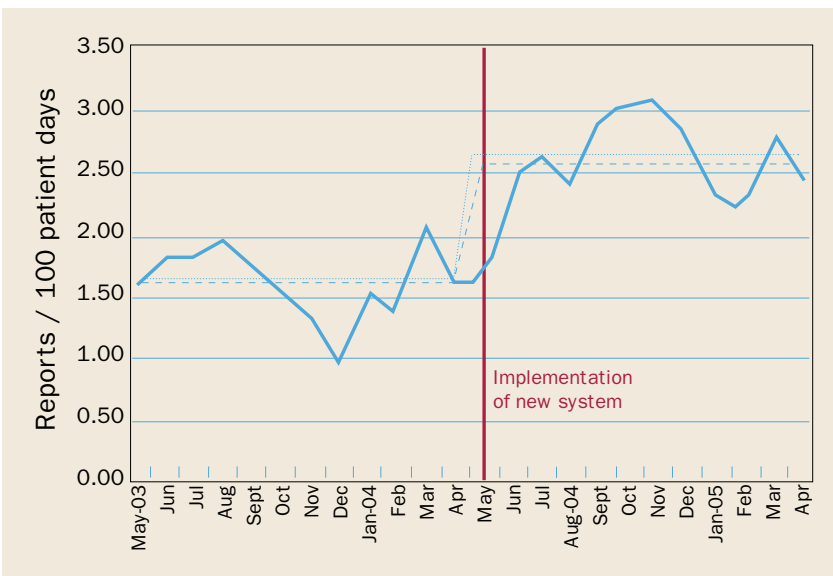

Figure 4 . Safety reporting system results $-\%$ potential (near miss) reports

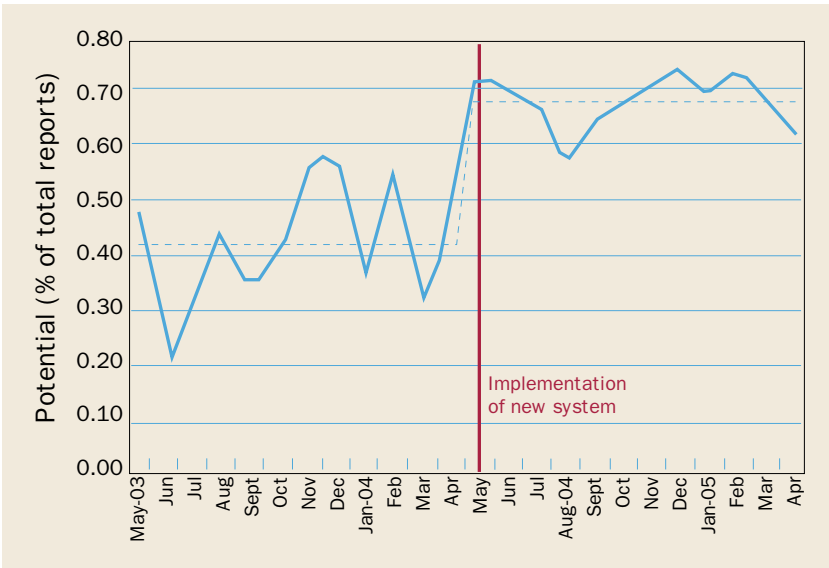

Figure 5. Logo for Sick Kids Partners in Patient Safety (PIPS) program
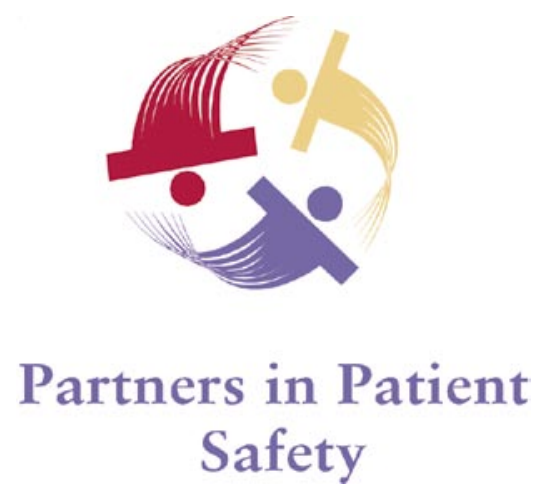
In addition to the safety reporting system, there are numerous other internal sources of safety information, including morbidity and mortality rounds, satisfaction surveys and other reviews and audits. The new quality and risk management database will be used to track follow-up of actions from these sources as well.

\section{Policies, Procedures and Guidelines}

Research into complex adaptive systems (such as hospitals) has shown that having a common purpose and easily understood rules can lead to innovative system behaviour (Committee on Quality Health Care in America 2001). Good procedures and guidelines can provide clarity in situations where there is expert agreement about the appropriate course of action, and they can provide useful learning tools for less experienced staff. These tools, however, need to be kept up-to-date and accessible to staff.

At Sick Kids, a complete review of our policies and procedures was required as there were no standards for policy-creation, and approval processes were ambiguous at best. Policies, procedures and guidelines existed that were out of date and in conflict with one another, and distribution, communication, retrieval and archiving of policies were also concerns. Further, in two serious adverse events, it was determined that the lack of accessible guidelines for the management of a particular group of complex patients was a contributing factor. In both cases, it was noted that good evidence-based guidelines had been developed; however, they had not been formally approved and were not available to the staff who required them.

The hospital's e-mail database system was selected to provide on-line access to policies by all staff, and to support search capabilities and electronic approval and review processes. To facilitate timely implementation, all existing hospital-wide policies, procedures and guidelines (unless they were deemed

\section{Table 4. Patient safety learning opportunities}

Key lessons in patient safety at orientation

Regular news items in the hospital's weekly newsletter

"Branding" of the patient safety program - called Partners in Patient Safety (PIPS), including a logo (Figure 5) depicting the relationship between staff, families and patients in improving safety, and formal launch during patient safety week last fall

Publication of a quarterly PIPS newsletter

Monthly patient safety rounds

Ad hoc area specific rounds and meetings

Patient safety Web site and resources

Quarterly meetings of area quality representatives featuring new hospital-wide initiatives and team successes and lessons learned clearly problematic) were migrated on to the new system, short review cycles were set and paper-based manuals and documents were removed from units and departments. Since implementation, more than 500 policies have been reviewed and updated, and approximately 300 have been permanently archived. Most recently, about 300 medical directives have also been added to the database and various departments are adding departmentbased documents to the system. Tighter controls have also been implemented to ensure that any new documents have supporting evidence, are developed with a hospital-wide perspective in mind and in consultation with appropriate stakeholders, and communication and evaluation plans have been developed. Each year, a selection of policies, procedures and guidelines undergo a formal audit. Some of the documents recently audited include introduction to innovative surgical procedures and devices, verbal orders and patient identification.

Currently, a large number of policies reside in draft mode and work continues to shorten the length of time between when a document is drafted to when it is approved and available to staff.

\section{Staff Education and Partnerships}

Healthcare workers need to know their role in providing safe care to patients and require education on general patient safety topics, area-specific safety initiatives and lessons learned elsewhere in the hospital and beyond.

In addition to informal mechanisms, such as one-on-one and team support, a number of formal opportunities for staff to learn about patient safety, to share "lessons learned" and to celebrate successes have been established and are listed in Table 4.

Staff also work with external partners to improve patient safety. The Blueprint has been shared in a number of external fora and was identified as a "good practice" in our recent

\section{Table 5. Feedback from Children's Council on patient safety}

Make sure playrooms are safe

Make sure kids in infectious diseases clinic follow infectioncontrol precautions

Make sure little kids cannot strangle on IV tubing

Provide lockers for families with a padlock like the kind at a fitness gym

Check often on kids who are alone in patient rooms to make sure they are safe

Improve the lighting in the parking garage

Cars double parked or stopped on the drive way make it unsafe

No smoking on property and especially around entrances to the hospital 
accreditation with the Canadian Council on Health Services Accreditation. Sick Kids has been invited to participate in government and hospital association planning regarding patient safety in hospitals. Sick Kids staff have also participated in teaching patient safety at learning institutions, and are active participants in the Canadian Association of Paediatric Health Centres (CAPHC) patient safety collaborative. A symposium titled "Partners in Paediatric Patient Safety: Taking Care of the Kids" has recently been coordinated by hospital staff.

\section{Partnering with Patients and Families}

Patients and families play an important role in ensuring safe care. They represent an important line of defense and should be encouraged to question organizational routine, procedures and processes and whenever something does not look or seem "right" (Agency for Healthcare Research and Quality 2001).

At Sick Kids, a team of parents and staff has recently been established called the "Families as Partners in Patient Safety" working group with the goals of raising awareness among healthcare professionals on the role of parents in patient safety, empowering family members to speak up and providing education to families about patient safety. One of the group's first activities was to ask members of the Children's Council their thoughts on making the hospital safer. Table 5 summarizes their feedback and underscores the value of listening to our young patients.

\section{Program Coordination}

The Department of Quality and Risk Management has primary responsibility for coordinating the patient safety plan, and, in February 2004, the role of Physician Liaison, Patient Safety was developed to enhance coordination and communication of patient safety throughout the organization. Direction has also been communicated to the program and department quality management leaders in regards to their role in patient safety, and plans are in place to enhance accountability for quality and safety through the development of regular reports and mandatory program elements. A major committee restructuring effort is currently underway to improve the value of committee work and to ensure alignment with hospital objectives including patient safety.

\section{Proactive Risk Assessment and Audit}

Risk assessment is the process of identifying processes and practices with either a high severity or high probability for patient harm. The Joint Commission on Accreditation of Healthcare Organizations (JCAHO) in the United States has said that "proactive identification and management of potential risks to patient safety has the obvious advantage of preventing adverse occurrences, rather than simply reacting when they occur. This approach also avoids the barriers to understanding created by hindsight bias and the fear of disclosure, embarrassment, blame, and punishment that can arise in the wake of an actual event" (JCAHO 2000).

With input from stakeholders throughout Sick Kids, an annual system-wide safety assessment is completed, which identifies a number of potential areas of focus. This year, the process for selection has been more formally developed (Figure 6), and an extensive list of audits and projects has been developed. Projects that are currently underway are listed in Table 6.

Past audits have included equipment maintenance processes, fridges and freezers, patient falls, referral processes to ambulatory clinics, and sedation practices and documentation. Other audits are currently underway and include timeliness of the first dose of antibiotics and our "responsible physician" policy, an important component to ensuring coordinated care.

An improved method for tracking the progress of projects and recommendations arising from audits is in development.

\section{Table 6. Current patient safety projects}

Correct site procedure

Critical laboratory tests

Entanglement / entrapment

Medication safety - heparin

Medication safety - high potency electrolytes

Medication safety - opioids

Medication safety - reconciliation

Patient identification

Prevention of central line infections

Prevention of surgical site infections

Transfer checklist roll-out

\section{Evaluation and Research}

All new safety projects are implemented with plans for their subsequent evaluation, including, if appropriate, plans for dissemination and publication of results. In the last few years, posters and presentations on various aspects of the plan have been presented at various conferences including CAPHC, the Ontario Hospital Association (OHA), the National Association of Children's Hospitals and Related Institutions (NACHRI), the National Initiative for Children's Healthcare Quality (NICHQ) and the Child Health Corporation of America (CHCA).

Patient safety, particularly in the paediatric environment, represents a relatively new area of study. At Sick Kids, a Patient Safety Research Interest Group has been formed to provide a venue for interchange of ideas and collaboration among Sick Kids staff. Research projects currently being developed include 
Building from the Blueprint for Patient Safety at the Hospital for Sick Children Polly Stevens, Anne Matlow and Ronald Laxer

Figure 6. Model for organizational patient safety, quality improvement and risk management priority-setting and planning

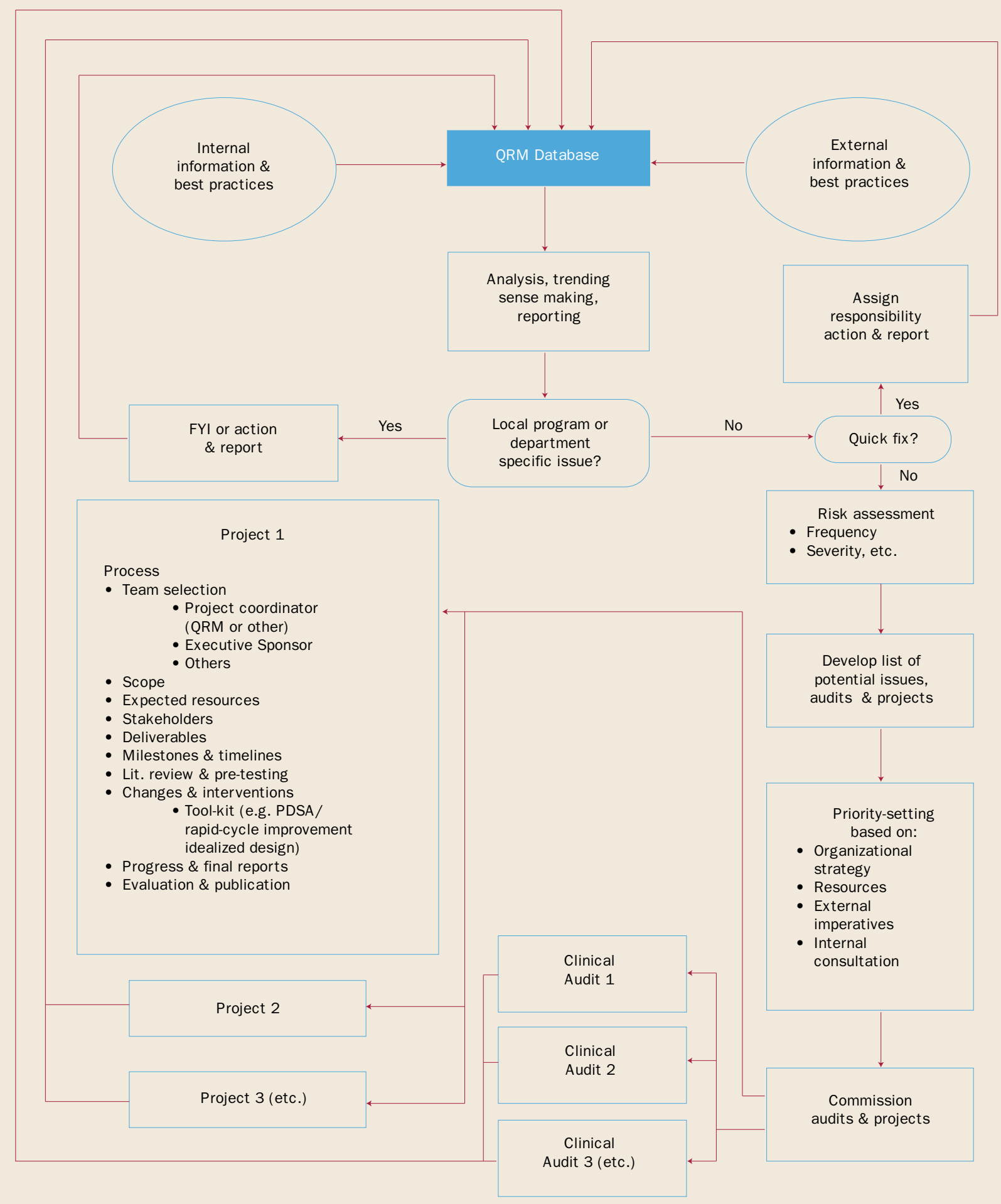


patient safety issues in ambulatory clinics and best practices in managing complex patients in the complex hospital environment. In conjunction with CAPHC, staff have also been instrumental in the development of a tool to examine the incidence of adverse events in hospitalized children in Canada.

Recently, Sick Kids championed a 10-centre CHCA study to enhance communication at patient hand-offs from the emergency department. The study found that by using a carefully designed checklist, significant improvements in medication management, duplication of laboratory tests and isolation precautions could be achieved. Plans are now in place to roll out the checklist to other areas of the organization, as well as to look for application of the concept to transfers between institutions.

\section{CONCLUSION}

The Blueprint for Patient Safety has provided us with a solid foundation for building our patient safety program. The 10 components of the program have served as a comprehensive framework for improving our safety culture, and for providing staff with insights into the many dimensions of patient safety. We consider the Blueprint to be a dynamic document, which will continue to grow and evolve over time as we move closer to our goal of providing safe care to every child who comes to us for care.

\section{References}

Agency for Healthcare Research and Quality. 2001. Five Steps to Safer Health Care. Rockville, MD: Author.

Bogner, M.S. 1994. Human Error in Medicine. Hillsdale, NJ: Lawrence Erlbaum Associates, Inc.

Chief Coroner of Ontario. 2002. Report on the Inquest of Sanchia Bulgin. Toronto, ON: Author.

Committee on Quality Health Care in America. 2001. Institute of Medicine. Crossing the Quality Chasm: A New Health System for the 21st Century. Washington, DC: National Academy Press.

Joint Commission on Accreditation of Healthcare Organizations (JCAHO). 2000. Patient Safety Standards. Oakbrook Terrace, IL: Author.

Kohn, L.T., J.M. Corrigan and M.S. Donaldson. 2000. To Err Is Human: Building a Safer Health System. Washington, DC: National Academy Press.

Kraman, S.S. and G. Hamm. 1999. "Risk Management: Extreme Honesty May Be the Best Policy." Annals of Internal Medicine 131(12): 963-67.

Marx, D. 2001. Patient Safety and the "Just Culture": A Primer for Health Care Executives. New York, NY: Columbia University.

Matlow, A., P. Stevens, C. Harrison and R. Laxer. 2004. "Achieving Closure Through Disclosure: Experience in a Pediatric Institution." Journal of Pediatrics 144(5): 559-60.

Reason, J.T. 1997. Managing the Risks of Organizational Accidents. Aldershof, UK: Ashgate.

Reason, J.T. 2000. "Human Error: Models and Management.” British Medical Journal 320(7237): 768-70.
Vincent, C., ed. 2001. Clinical Risk Management: Enhancing Patient Safety. 2nd ed. London, UK: BMJ Books.

Weick, K.E. and K.M. Sutcliffe. 2001. Managing the Unexpected: Assuring High Performance in an Age of Complexity. San Francisco, CA: Jossey-Bass.

Zimmerman, B., C. Lindberg and P. Plesk. 1998. Edgeware: Insights from Complexity Science for Health Care Leaders. Irving, TX: VHA Inc.

Zipperer, L.A. and S. Cushman, eds. 2001. Lessons in Patient Safety. Chicago, Ill: National Patient Safety Foundation.

\section{About the Authors \\ Polly Stevens, MHSc, is Director, Quality and Risk Management, The Hospital for Sick Children.}

Anne Matlow, MD, FRCPC, is Physician Liaison, Patient Safety and Director, Infection Prevention and Control Program, The Hospital for Sick Children.

Ronald Laxer, MD, FRCPC, is Vice-president, Clinical and Academic Affairs, The Hospital for Sick Children.

Corresponding Author: Polly Stevens, Director, Quality and Risk Management, The Hospital for Sick Children, 555 University Avenue, Toronto, Ontario M5G 1X8, Tel: (416) 813-5357, polly. stevens@sickkids.ca

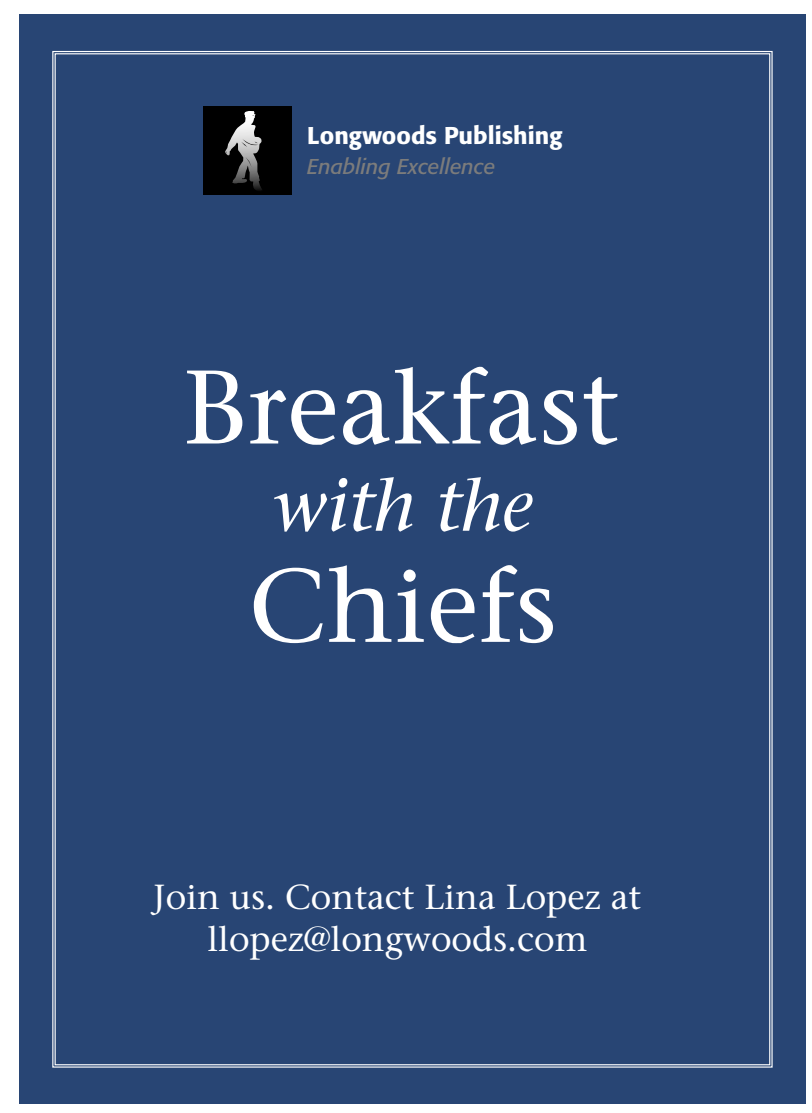

